\title{
Co-prescription of co-trimoxazole and spironolactone in elderly patients
}

\author{
The combination should be used with caution because of the risk of hyperkalaemia
}

\author{
Li Wei lecturer, Thomas M MacDonald professor, Isla S Mackenzie clinical senior lecturer
}

Medicines Monitoring Unit (MEMO) and Hypertension Research Centre (HRC), Division of Medical Sciences, University of Dundee, Ninewells Hospital and Medical School, Dundee DD1 9SY, UK

\begin{abstract}
Although much effort goes into understanding the risks and benefits of recently licensed drugs, less is known about many of the older drugs that are prescribed widely. For example, the phrase "safe as aspirin" is often used to extol the safety virtues of a drug, even though aspirin is one of the main culprits when it comes to hospital admissions related to an adverse drug event. ${ }^{1}$ Spironolactone and trimethoprim-sulfamethoxazole (co-trimoxazole) are two "older" drugs about which we should know more. In the linked case-control study (doi:10.1136/bmj. d5228), Antoniou and colleagues assess the risk of admission to hospital for hyperkalaemia in elderly patients treated with co-trimoxazole in combination with spironolactone. ${ }^{2}$
\end{abstract}

Spironolactone works well in congestive heart failure, ${ }^{3}$ resistant hypertension, ${ }^{4}$ hyperaldosteronism, ${ }^{5}$ and hypertension in general. ${ }^{6}$ Despite hypertension being an unlicensed indication in the United Kingdom, the new guidelines from the National Institute for Health and Clinical Excellence (NICE) on hypertension name spironolactone as an option for fourth line treatment. ${ }^{7}$ Because of these benefits, the use of spironolactone in the UK and elsewhere has increased greatly over recent years, mainly for the above indications, but also for secondary aldosteronism resulting from cirrhotic liver disease. ${ }^{8}$

The down side of spironolactone is the risk of hyperkalaemia, which is increased in older people; those with renal impairment, diabetes, or a high pretreatment serum potassium; and when it is co-administered with potassium supplements and drugs that reduce potassium excretion. Hyperkalaemia with spironolactone has been reported in patients with congestive heart failure, ${ }^{9}$ but this has not been a major problem in the UK because of the cautious prescribing and frequent monitoring of renal function within the NHS. ${ }^{8}$ The more selective aldosterone antagonist, eplerenone, may be less toxic than spironolactone, but it is also less effective, at least in patients with hypertension and aldosteronism. ${ }^{10}$

In Canada, co-trimoxazole is the most commonly prescribed antibiotic for urinary tract infection. This is unlike the UK, where the use of co-trimoxazole is restricted because of concerns about serious cutaneous adverse reactions and blood dyscrasias. In the UK, trimethoprim, a component of co-trimoxazole, is much more commonly used for this indication. Hyperkalaemia is also a risk with co-trimoxazole, probably because of the potassium sparing action of trimethoprim.
Given the benefits and potential for toxicity with spironolactone and trimethoprim or co-trimoxazole, any new data that will inform the risk-benefit balance of these drugs are welcome. Antoniou and colleagues' study shows an important adverse pharmacodynamic interaction between co-trimoxazole and spironolactone in elderly patients that can lead to hospital admission for hyperkalaemia — a relatively "hard" biochemical end point. There was also a smaller risk of hyperkalaemia with co-prescription of nitrofurantoin. The same authors have previously reported serious hyperkalaemia associated with co-prescription of co-trimoxazole and angiotensin converting enzyme inhibitors or angiotensin receptor blockers (adjusted odds ratio $6.7,95 \%$ confidence interval 4.5 to 10.0 ), which is thought to be caused by a similar pharmacodynamic interaction. ${ }^{11}$

In the current study, Antoniou and colleagues reported that patients taking spironolactone and co-trimoxazole were about 12 times more likely to be admitted to hospital for hyperkalaemia (12.4, 7.1 to 21.6$)$ than those taking spironolactone with amoxicillin. ${ }^{2}$ The risks of co-trimoxazole were also dose related. The population attributable fraction suggests that around $60 \%$ of admissions to hospital for hyperkalaemia in elderly patients taking spironolactone and an antibiotic for a urinary tract infection could be avoided if co-trimoxazole was not co-prescribed with spironolactone.

As with all observational studies, various factors might confound the outcomes of interest because of the co-linearity of some variables in the study. However, the greatly increased risk (around 12-fold) suggests that minor problems with data adjustments would not have changed the overall finding of an increased risk of hyperkalaemia for co-prescribing co-trimoxazole and spironolactone.

To set the results in a UK context, trimethoprim is prescribed to about 60 per 1000 of the UK population each year, co-trimoxazole to 0.8 per 1000 , nitrofurantoin to 18 per 1000 , and spironolactone to 7 per 1000 (data for 2010 from the MEMO database $^{12}$ ). The co-prescription of spironolactone with trimethoprim is therefore likely to occur infrequently but is set to increase as spironolactone is used more often and broad spectrum antibiotics for urinary tract infection are avoided to mitigate the development of Clostridium difficile colitis.

We need to know if trimethoprim alone will cause a similar adverse drug interaction as co-trimoxazole (as seems likely), 
whether these risks extend to younger people, and whether other factors increase the risks. So, as always, more research is needed. In the interim, a cautious approach is needed and the use of alternative antibiotics should be considered where appropriate. Monitoring for hyperkalaemia should be increased, and temporarily reducing the dose of spironolactone or angiotensin converting enzyme inhibitors or angiotensin receptor blockers might be useful in patients receiving these drugs in combination with trimethoprim, co-trimoxazole, or even (until further data are available) nitrofurantoin.

Competing interests: All authors have completed the ICMJE uniform disclosure form at www.icmje.org/coi_disclosure.pdf (available on request from the corresponding author) and declare: no support from any organisation for the submitted work; no financial relationships with any organisations that might have an interest in the submitted work in the previous three years; MEMO receives grant funding from the drug industry to carry out drug safety studies but we have no conflicts of interest in relation to the current article.

Provenance and peer review: Commissioned; not externally peer reviewed.

Pirmohamed M, James S, Meakin S, Green C, Scott AK, Walley TJ, et al. Adverse drug reactions as cause of admission to hospital: prospective analysis of 18820 patients. BMJ 2004;329:15-9.
2 Antoniou T, Gomes T, Mamdani MM, Yao Z, Hellings C, Garg AX, et al. Trimethoprim-sulfamethoxazole induced hyperkalaemia in elderly patients receiving spironolactone: nested case-control study. BMJ 2011;343:d5228.

3 Pitt B, Zannad F, Remme WJ, Cody R, Castaigne A, Perez A, et al. The effect of spironolactone on morbidity and mortality in patients with severe heart failure. Randomized Aldactone Evaluation Study investigators. N Engl J Med 1999;341:709-17.

4 Nishizaka MK, Zaman MA, Calhoun DA. Efficacy of low-dose spironolactone in subjects with resistant hypertension. Am J Hypertens 2003;16:925-30.

5 Lim PO, Young $\mathrm{W} \mathrm{Jr}$, MacDonald TM. A review of the medical treatment of primary aldosteronism. $J$ Hypertens 2001;19:353-61.

6 Parthasarathy HK, Alhashmi K, McMahon AD, Struthers AD, McInnes GT, Ford I, et al. Does the ratio of serum aldosterone to plasma renin activity predict the efficacy of diuretics in hypertension? Results of RENALDO. J Hypertens 2010;28:170-7.

7 National Institute for Health and Clinical Excellence. Hypertension: clinical management of primary hypertension in adults. CG127. 2011. http://guidance.nice.org.uk/CG127/ Guidance/pdf/English.

8 Wei L, Struthers AD, Fahey T, Watson AD, Macdonald TM. Spironolactone use and renal toxicity: population based longitudinal analysis. BMJ 2010;340:c1768.

9 Juurlink DN, Mamdani MM, Lee DS, Kopp A, Austin PC, Laupacis A, et al. Rates of hyperkalemia after publication of the Randomized Aldactone Evaluation Study. N Engl J Med 2004;351:543-51.

10 Parthasarathy HK, Ménard J, White WB, Young WF Jr, Williams GH, Williams B, et al. A double-blind, randomized study comparing the antihypertensive effect of eplerenone and spironolactone in patients with hypertension and evidence of primary aldosteronism. $J$ Hypertens 2011;29:980-90.

11 Antoniou T, Gomes T, Juurlink DN, Loutfy MR, Glazier RH, Mamdani MM. Trimethoprim-sulfamethoxazole-induced hyperkalemia in patients receiving inhibitors of the renin-angiotensin system: a population-based study. Arch Intern Med 2010;170:1045-9.

12 Wei L, MacDonald TM. The Tayside Medicines Monitoring Unit (MEMO). In: Strom BL, ed. Pharmacoepidemiology. 4th ed. John Wiley, 2005:323-36.

Cite this as: BMJ 2011;343:d5656

(c) BMJ Publishing Group Ltd 2011 